\title{
A ADOÇÃO DO LIVRO DIDÁTICO ELETRÔNICO PARA DISPOSITIVOS MÓVEIS NA EDUCAÇÃO SUPERIOR A DISTÂNCIA: MOTIVAÇÕES, DIFICULDADES E ESTRATÉGIAS DE CONTORNO
}

\author{
Ana Luisa Mülbert - Universidade do Sul de Santa Catarina - amulbert@ gmail.com
}

Alice T. Cybis Pereira - Universidade Federal de Santa Catarina - acybis@gmail.com

\begin{abstract}
Resumo
Este artigo apresenta os resultados do processo de implementação e avaliação de um livro didático eletrônico (ebook) adaptado para dispositivos móveis, adotado no contexto da educação superior a distância. Na avaliação da iniciativa foram diagnosticadas as motivações e dificuldades vivenciadas pelos estudantes no uso da mídia, a partir das quais identificou-se possíveis estratégias de contorno das dificuldades. Este trabalho foi desenvolvido alinhado à abordagem de Pesquisa de Desenvolvimento aplicada à área de educação (Design-Based Research), orientada à busca de soluções práticas e inovadoras para problemas educacionais. A experimentação prática desenvolvida alcançou 1.836 estudantes, dos quais 223 responderam ao questionário de avaliação. Os resultados apontam para uma percepção positiva da mídia, mas também para resistências de adoção em certas circunstâncias.
\end{abstract}

Palavras-chave: Livro didático eletrônico. Ebook. EPUB. M-learning. Educação a distância.

\begin{abstract}
This paper presents the outcomes of the implementation and evaluation process of an electronic textbook, adapted to mobile devices, adopted in the context of distance higher education. In the evaluation step of the initiative, motivations and difficulties expressed by students that used the media were diagnosed, from which possible strategies to overcome the difficulties were identified. This work was developed aligned to the Design-Based Research approach, oriented to the research on practical and innovative solutions of educational problems. The practical experimentation developed reached 1,836 students, of which 223 answered to the evaluation questionnaire. The results point to a positive perception of etextbooks' use in the students' mobile devices, but also point to adoption resistances in some circumstances.
\end{abstract}

Keywords: E-textbook. Ebook. EPUB. M-learning. Distance learning. Educational resources.

\section{Introdução}

As atividades humanas contemporâneas e a interação social dos indivíduos têm sido significativamente afetadas por um ambiente de computação ubíqua e sem fio, proporcionando novos hábitos e ritmos à vida social, marcados pela mobilidade, novas formas de usar os espaços públicos e novas possibilidades de explorar fontes de informação e comunicação (NEW MEDIA CONSORTIUM, 2013). Segundo Santaella (2010), as práticas proporcionadas pelas novas mídias e tecnologias estão construindo um novo espaço de misturas inextricáveis entre o virtual (ciberespaço) e os ambientes físicos que nossos corpos habitam, formando assim espaços híbridos. Tais espaços são também móveis, se constituem em múltiplos espaços em um mesmo espaço físico e desafiam os sentidos de localização, permanência e duração.

A ampliação do acesso e disponibilidade de tecnologias móveis tem favorecido a emergência de uma alternativa educacional que vem sendo denominada na literatura científica de m-learning (ou mobile learning), que consiste no uso educacional de dispositivos móveis e portáteis em atividades de ensino e aprendizagem. Cada vez mais o estudante pode ter acesso 
a uma multiplicidade de diferentes dispositivos portáteis e está imerso em um meio físico e digital que provê uma infraestrutura de apoio, que favorece o deslocamento pelo espaço físico sem perder a conexão com atividades e pessoas (SHARPLES et al., 2007; TRAXLER, 2009).

Neste contexto, em programas de educação a distância (EAD), surge o desafio de conceber os recursos didáticos também adaptados ao uso por meio das tecnologias móveis. Um dos recursos didáticos comumente utilizado na EAD é o livro didático, frequentemente disponibilizado em mídia impressa ou em seu equivalente em mídia digital para uso no computador. Esse recurso vem sendo estendido também para uso em dispositivos móveis, contudo, segundo Arenas e Barr (2013), em movimentos como esse não se trata apenas de duplicar o livro impresso para um dispositivo móvel, mas principalmente de redefini-lo.

Um dos formatos que vem se consolidando para a publicação de ebooks ou livros eletrônicos ${ }^{1}$ é o EPUB (Electronic Publication), que corresponde ao formato digital capaz de representar, empacotar e codificar conteúdos web para distribuição consolidada em um único arquivo. Este padrão possibilita a produção e distribuição de um conteúdo fluido, rico e operável em múltiplas plataformas, capaz de se adaptar aos atributos da tela do dispositivo que o contém (INTERNATIONAL DIGITAL PUBLISHING FORUM, 2014).

Mas o amadurecimento dos padrões tecnológicos e a ampla disseminação das tecnologias móveis não parece ser ainda suficiente para garantir a adoção do livro didático eletrônico (LDE) como um recurso de apoio efetivo ao processo de aprendizagem. Por isso, nesse artigo apresenta-se um diagnóstico sobre as motivações e dificuldades de estudantes de graduação para a adoção do LDE em dispositivos móveis. Aos estudantes foi criada a oportunidade de uso de um ebook especialmente desenvolvido para uma disciplina de graduação em EAD. A oferta deste novo recurso ocorreu de modo concomitante com o livro impresso e a versão imprimível em PDF, de modo a deixar ao estudante a escolha pela mídia desejada. Essa experiência foi avaliada pelos estudantes que relataram, entre outros aspectos, seus motivos e dificuldades para usar ou não o LDE em seus dispositivos móveis. Os partir dos resultados diagnosticados, apresenta-se também possíveis estratégias institucionais de contorno das dificuldades e obstáculos encontrados.

\section{O contexto da pesquisa}

Este estudo foi desenvolvido no Campus Virtual da Universidade do Sul de Santa Catarina (Unisul), que é uma universidade comunitária que oferta vários cursos e disciplinas a distância. O desenho metodológico de EAD adotado pela instituição é constituído por um conjunto de estratégias e recursos pedagógicos, associados à ação mediadora de tutores e professores por meio de ambiente virtual de aprendizagem (AVA). A grande maioria dos recursos didáticos utilizados são produzidos na própria instituição, entre os quais estão os livros didáticos impressos e online, os objetos multimídia, as videoaulas gravadas e as atividades e avaliações. Com exceção do livro impresso, todos os demais recursos são entregues ao estudante pelo AVA. Para a experimentação relatada neste artigo desenvolveu-se alguns recursos a mais, como o LDE em formato EPUB e outras mídias acessórias para divulgar e favorecer a disseminação da mídia entre os estudantes.

\section{Aspectos metodológicos}

Este trabalho foi desenvolvido alinhado à abordagem de pesquisa chamada na língua inglesa de Design-Based Research (DBR) e que, no Brasil, Matta et al. (2014) propõem denominar de Pesquisa de Desenvolvimento em educação ${ }^{2}$. Essa abordagem tem sido especialmente adotada

\footnotetext{
${ }^{1}$ Em português, observa-se o uso corrente de termos como "livro digital", "livro eletrônico" ou ainda do termo ebook, sem tradução. Adotou-se neste trabalho a denominação de "livro eletrônico".

2 Para compreender detalhes sobre a DBR pode-se consultar a documentação de Design-Based Research Collective (2003).
} 
para apoiar investigações com foco no desenvolvimento de soluções práticas e inovadoras para problemas da educação. O processo de pesquisa da DBR, com forte ênfase pragmática, consiste de múltiplas iterações em busca de uma solução que em repetidos ciclos vai se refinando (ANDERSON e SHATTUCK, 2012), Assim, a pesquisa aqui apresentada se desenvolveu em várias etapas: (i) discussões de um grupo de especialistas educacionais para delinear as primeiras características da intervenção e das características do LDE; (ii) o desenho e construção do LDE em formato EPUB para uso em dispositivos móveis ; (iii) a oferta desta mídia em um contexto real de EAD, seguida pela (iv) avaliação da experiência pelos estudantes.

A oferta do LDE ocorreu em uma disciplina a distância, com 1.836 estudantes matriculados em diversas turmas. O levantamento de dados com os estudantes foi realizado por meio de um questionário de pesquisa online, com questões objetivas e abertas. Os dados qualitativos foram tratados e codificados com o apoio do software Atlas.ti de tal modo que, em um conjunto de dados inicialmente pouco estruturados, foi possível identificar e selecionar partes importantes, padrões repetidos e fazer algumas associações entre eles. Dados quantitativos e qualitativos foram articulados entre si para, em uma ação interpretativa, extrair o sentido das percepções dos estudantes sobre a iniciativa.

Quadro 1 - Características estudantes que responderam o questionário de avaliação

\begin{tabular}{|l|c|c|l|c|c|}
\hline Modalidade do curso de origem & Num. & $\mathbf{\%}$ & Sistema operacional do dispositivo & Num & $\%$ \\
\hline Presencial & 131 & $59 \%$ & Android & 106 & $48 \%$ \\
\hline A distância & 88 & $39 \%$ & iOS & 87 & $39 \%$ \\
\hline Não identificado & 4 & $2 \%$ & Outros & 8 & $4 \%$ \\
\hline Propriedade do dispositivo & Num. & $\%$ & Outros dados & Num & \\
\hline Possui somente smartphone & 126 & $57 \%$ & Num. de usuários do livro eletrônico & 73 & \\
\hline Possui tablet e smartphone & 63 & $28 \%$ & Cursos de origem dos estudantes & 28 & \\
\hline Não possui tablet nem smartphone & 22 & $10 \%$ & Polos de origem dos estudantes de EAD & 40 & \\
\hline Possui somente tablet & 12 & $5 \%$ & Estados de origem dos estudantes & 16 & \\
\hline
\end{tabular}

Dentre o público alvo, 223 estudantes responderam o questionário, dos quais 73 afirmaram ter feito efetivo uso do LDE. Uma seção adicional do questionário foi respondida exclusivamente por aqueles que fizeram uso do LDE, e os dados de todos os respondentes foram utilizados para diagnosticar dificuldades e sugestões. O Quadro 1 sintetiza as características dos participantes da coleta de dados, que representam um grupo heterogêneo, com estudantes originários de múltiplos cursos, residentes em variados locais, vinculados a cursos de graduação de diferentes modalidades e com variados graus de acesso e uso das tecnologias móveis.

\section{Ortefato: livro didático eletrônico para dispositivo móvel}

$\mathrm{O}$ artefato desenvolvido resultou da transformação de um conteúdo preexistente como livro impresso, sobre o qual foi necessário fazer várias alterações para adequação ao novo meio de acesso. Os atributos implementados contemplaram três aspectos principais: a adaptabilidade do conteúdo ao dispositivo, a navegabilidade no livro e a inserção de links de acesso a fontes externas ao livro. Não foram implementados recursos mais avançados, como por exemplo a incorporação de vídeos ou de outros recursos embarcados acessíveis quando o dispositivo está desconectado da internet. Essa decisão resultou do desejo de obter uma mídia de formato leve, com conteúdos adequados a dispositivos com dimensões mais reduzidas, para com isso 
contemplar um maior número de modelos de dispositivos e ampliar o alcance da iniciativa a um maior grupo de estudantes. A Figura 1 ilustra o artefato implementado.

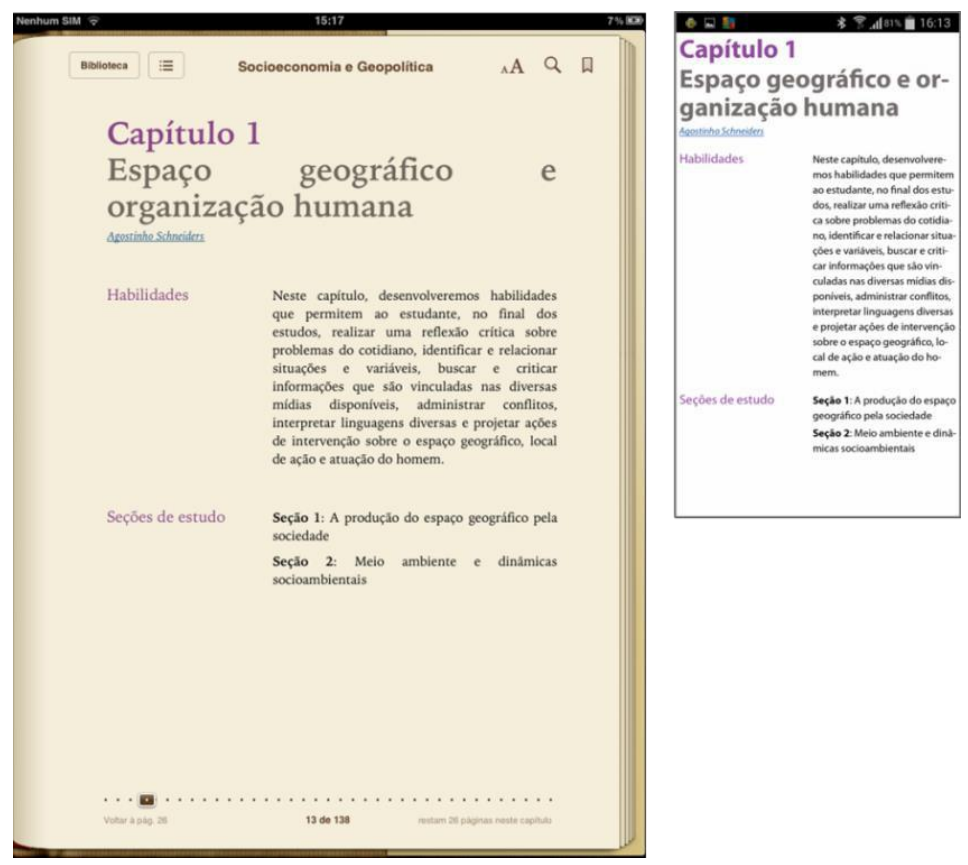

Figura 1 - Telas com a mesma página do livro eletrônico visualizadas em diferentes dispositivos (tablet com iOS e smartphone com Android)

\section{Motivações e benefícios percebidos no uso do livro eletrônico}

Um dos pressupostos desta pesquisa é de que o livro eletrônico poderia vir a ser um recurso gerador de benefícios aos estudantes. As manifestações dos estudantes permitiram compreender melhor esse fenômeno, qualificando melhor os benefícios percebidos e as circunstâncias em que eles se tornam atraentes. Nas falas dos estudantes observou-se diversas manifestações que revelam que o livro eletrônico é um recurso que pode trazer benefícios:

\footnotetext{
Facilitam a vida do estudante, que durante as horas vagas, pode fazer uso destes em qualquer lugar.
}

Acredito que os dispositivos móveis só venham a acrescentar nos estudos

Segundo Liu et al. (2010), a percepção positiva dos estudantes sobre a facilidade e a utilidade da tecnologia móvel, bem como o grau de esforço envolvido em seu uso, influencia sua intenção de utilizar a tecnologia móvel nos estudos. Por isso, no questionário foram apresentadas questões para verificar se o livro eletrônico é percebido como um recurso que pode contribuir para facilitar os estudos, para melhorar o desempenho ou exigir maior esforço para estudar. Na Figura 2 observa-se que a maioria dos estudantes considera o livro eletrônico como um recurso facilitador, capaz de contribuir para a melhoria do desempenho. Nota-se, contudo, que em relação ao esforço exigido há um cenário de opiniões diversificadas. Como para avaliar a exigência de esforço é necessário ter experiência prévia, a inexperiência no uso do livro eletrônico em atividades reais de estudo formal pode ser um fator gerador de incertezas. Esta inexperiência foi revelada pelo diagnóstico (manifesto nas respostas de várias perguntas do questionário) de que muitos estudantes desconhecem os recursos do programa leitor (de anotações, destaques de texto, marcadores de página, conversão de texto para áudio, entre outros) e também pouco sabem discernir sobre as diferenças entre o formato EPUB e PDF. 


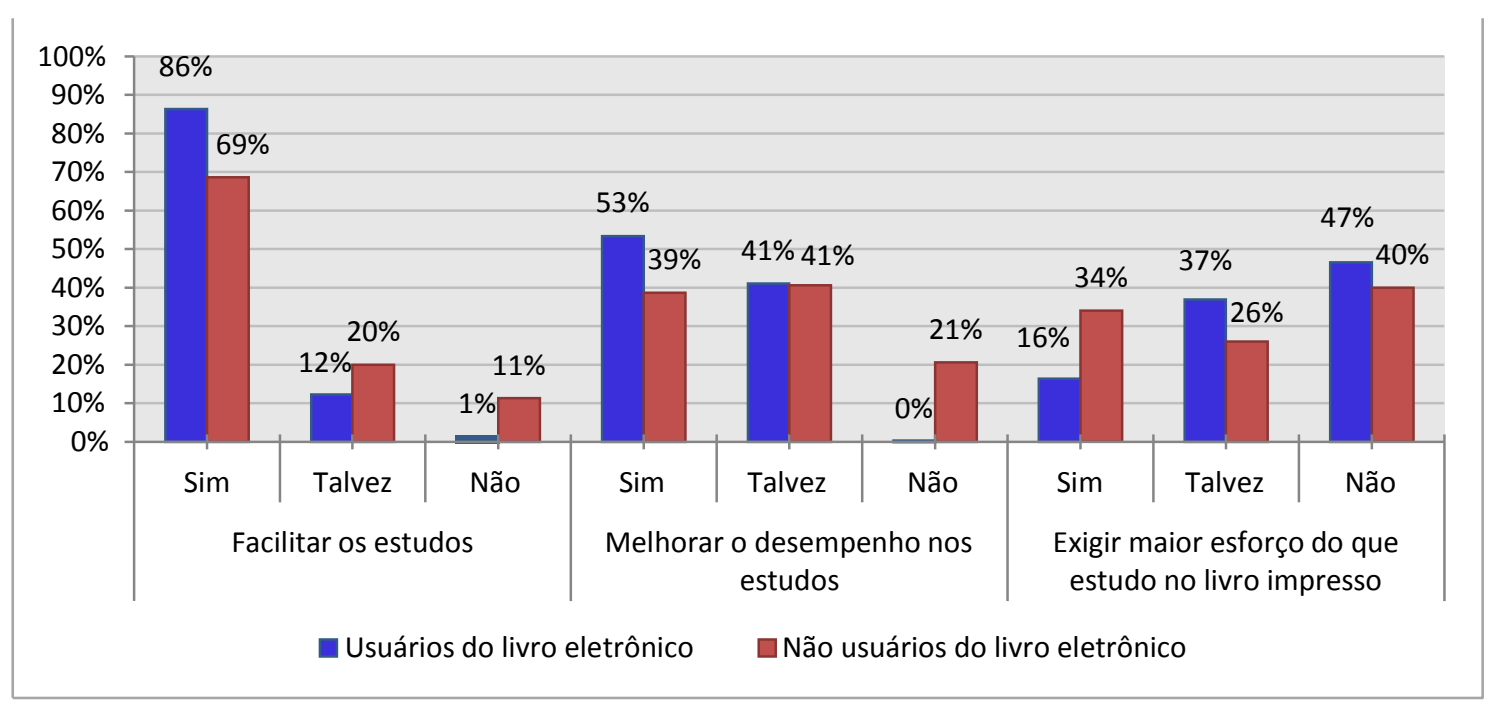

Figura 2 - Benefícios percebidos no uso do livro eletrônico, conforme tipo de estudante.

De modo geral, é visível que entre os estudantes que usaram o livro eletrônico há uma percepção mais positiva de que o livro eletrônico pode trazer facilidades ao estudo, mas benefícios como a melhoria do desempenho e principalmente o esforço demandado no uso são aspectos em que há ainda dúvidas expressivas. Nos dados qualitativos identificou-se também que fatores como uma possível redução nos custos dos estudos e questões ambientais relacionadas à redução do uso do papel podem ser fatores motivadores para uma parcela dos estudantes. As falas a seguir ilustram esse posicionamento:

Sem dúvidas se o material totalmente eletrônico pudesse diminuir o custo com mensalidade iria ajudar muito.

Acredito que agrupar o material de estudo em meu tablet seria de suma importância pois reduziria a quantidade de papéis a serem carregados, sem contar na economia que isso poderia trazer tanto para o meu bolso quanto para o meio ambiente.

Mas apesar de uma percepção predominantemente positiva sobre o LDE, emergiram também nos dados diversas manifestações de dificuldades, conforme relatado na próxima seção.

\section{As dificuldades dos estudantes}

Para investigar os obstáculos da iniciativa e os possíveis caminhos de solução, foram incluídas questões abertas que estimularam o registro de dificuldades percebidas na iniciativa. As respostas possibilitaram a categorização das dificuldades, ilustradas no Quadro 2. Entre as manifestações dos estudantes notou-se o interesse no livro eletrônico, desde que este não seja um substituto do livro impresso e que não exija a presença de recursos muito sofisticados nos dispositivos. Notou-se também o desejo dos estudantes de que a iniciativa não gere custos adicionais.

Percebeu-se que as manifestações dos estudantes primam pela manutenção do status quo, são defensivas e buscam garantir a permanência dos recursos e rotinas com as quais já estão habituados. Implícito está uma postura conservadora de certa resistência à mudança, na qual um novo recurso é aceito, desde que não desacomode hábitos já instalados. A fala de um dos estudantes ilustra esse movimento e destaca a necessidade de superação de hábitos arraigados: 
livros impressos, mas que após um certo período de adaptação, essa tarefa se torna mais fácil de ser executada, seja por obrigação ou por interesse e desejo de empenhar-se em ler um livro de formato PDF ou ebook

A autopercepção deste estudante e sua disponibilidade de desacomodação, contudo, não é uma regra predominante. Percebe-se, portanto, a necessidade de identificar mecanismos para lidar com as resistências e gerenciar as mudanças no caso de haver uma proposição institucional de adoção do novo recurso didático.

Quadro 2 - Classificação e descrição das dificuldades de uso do LDE pelos estudantes

\begin{tabular}{|c|c|c|}
\hline DIFICULDADES & DESCRIÇÃO & TIPO \\
\hline Interface limitada e desconforto & Limitações da tela e desconforto no uso prolongado. & Física \\
\hline Anotações no livro são difíceis & $\begin{array}{l}\text { Desconhecimento ou falta de hábito sobre como fazer anotações } \\
\text { sobre a mídia. }\end{array}$ & Física \\
\hline $\begin{array}{l}\text { Limitações dos recursos do } \\
\text { dispositivo (hardware) }\end{array}$ & $\begin{array}{l}\text { As características dos dispositivos dos estudantes são } \\
\text { incompatíveis com os recursos exigidos pela mídia. }\end{array}$ & Física \\
\hline $\begin{array}{l}\text { Impressão do livro pelo } \\
\text { estudante }\end{array}$ & $\begin{array}{l}\text { A substituição do livro impresso pelo eletrônico representa um } \\
\text { custo e trabalho adicional para quem quer imprimi-lo. }\end{array}$ & Física \\
\hline $\begin{array}{l}\text { Dispositivo é inapropriado para } \\
\text { interações ativas }\end{array}$ & $\begin{array}{l}\text { A interatividade nas ferramentas de mediação a distância é } \\
\text { dificultada quando realizada por dispositivos móveis. }\end{array}$ & Física \\
\hline Dispersão da atenção & $\begin{array}{l}\text { Dispersão da atenção decorrente da concorrência com outras } \\
\text { funções que o dispositivo também desempenha. }\end{array}$ & Cognitiva \\
\hline $\begin{array}{l}\text { Desconhecimento no uso do } \\
\text { programa leitor }\end{array}$ & $\begin{array}{l}\text { Pouco conhecimento sobre recursos avançados dos programas de } \\
\text { manipulação de ebooks (leitor). }\end{array}$ & Cognitiva \\
\hline $\begin{array}{l}\text { Dispositivos não costumam ser } \\
\text { usados para estudo }\end{array}$ & Falta de hábito de uso em atividades de aprendizagem. & $\begin{array}{l}\text { Socio- } \\
\text { cultural }\end{array}$ \\
\hline $\begin{array}{l}\text { AVA e outros recursos não } \\
\text { adaptados ou incompatíveis }\end{array}$ & $\begin{array}{l}\text { Dificuldades para usar outros recursos de EAD por meio do } \\
\text { dispositivo móvel, quando estes não foram adaptados. }\end{array}$ & Contextual \\
\hline Uso na avaliação presencial & $\begin{array}{l}\text { Avaliações com consultas são inviabilizadas devido a } \\
\text { impossibilidade de uso de dispositivo neste evento. }\end{array}$ & Contextual \\
\hline Conectividade limitada & $\begin{array}{l}\text { Recursos escassos de conectividade à internet que dificultam o } \\
\text { acesso á mídia e seus recursos embarcados. }\end{array}$ & Contextual \\
\hline $\begin{array}{l}\text { Desigualdade entre recursos dos } \\
\text { estudantes }\end{array}$ & $\begin{array}{l}\text { Características dos dispositivos dos estudantes são heterogêneas } \\
\text { e pouco padronizadas. }\end{array}$ & Contextual \\
\hline $\begin{array}{l}\text { Custo de propriedade do } \\
\text { dispositivo }\end{array}$ & $\begin{array}{l}\text { Custos de serviços de conexão e dos dispositivos avançados são } \\
\text { altos e limitam as possibilidades dos estudantes. }\end{array}$ & Contextual \\
\hline
\end{tabular}

Com exceção de um estudante que sugeriu a conversão do livro impresso para o formato de audiolivro, nota-se total ausência de sugestões a respeito dos atributos da mídia propriamente dita. Não se observou nenhuma sugestão quanto a recursos estéticos, de pesquisa ou outros atributos que poderiam ser incorporados ao livro eletrônico. Tal cenário faz sentido, e também reforça, a constatação sobre a pouca experiência que o estudante possui no uso de livros eletrônicos.

Girard (2014) classificou as barreiras no uso de ebooks em três categorias: físicas, cognitivas e socioculturais. As barreiras físicas são aquelas associadas com as expectativas dos indivíduos de ter recursos normalmente associados ao uso do texto impresso ou imprimível, como a possibilidade de anotar e marcar no livro ou de imprimi-lo e reproduzi-lo. A comparação entre o suporte em papel e o digital costuma ser a tônica das barreiras físicas. As barreiras cognitivas são aquelas que impedem ou limitam os processos mentais ou, ainda, as ideias contrárias ao uso do livro eletrônico. São barreiras relacionadas a aspectos da personalidade do usuário, gostos pessoais, habilidades cognitivas e de percepções quanto à utilidade e valor dos ebooks. As barreiras socioculturais, por sua vez, são aquelas que resultam de regras sociais e culturais que exercem influência sobre o indivíduo e que estão relacionadas ao momento e lugar onde a leitura acontece. 
Entre as dificuldades relatadas pelos estudantes pode-se identificar os três tipos de barreiras citadas. As barreiras físicas estão evidentes nas dificuldades sobre as limitações da interface do dispositivo e nas dificuldades de fazer anotações sobre o livro eletrônico. As barreiras cognitivas estão evidentes nas dificuldades de manter a atenção e a falta de conhecimento no uso do programa leitor. As barreiras de natureza sociocultural podem ser identificadas nas percepções de que os dispositivos não costumam ser usados na aprendizagem formal, ou nas manifestações de resistências à mudança dos hábitos de estudo. Há, contudo, outras dificuldades identificadas que não se enquadram na categorização de Girard (2014). Estas se referem às dificuldades de inserção do uso da mídia no contexto educacional ou ainda de aspectos de infraestrutura que extrapolam o ambiente educacional. São as dificuldades relacionadas ao uso do AVA por meio dos dispositivos, da integração do livro eletrônico aos demais recursos de EAD, da indisponibilidade de infraestrutura de telecomunicações, entre outras. Assim, identificou-se uma nova categoria de barreiras (contextual), que representa o conjunto de dificuldades relacionadas ao contexto em que o estudante está inserido.

No Quadro 2 as dificuldades relatadas pelos estudantes foram categorizadas conforme o tipo de barreira que representam. Essa tipologia foi utilizada para guiar a discussão e investigação na literatura por estratégias de contorno das dificuldades, conforme apresentado na próxima seção.

\section{$7 \quad$ O contorno das dificuldades pela ótica institucional}

Por parte das instituições educacionais, parece haver pouco a ser feito para eliminar as barreiras físicas e algumas barreiras contextuais que extrapolam o contexto educacional. Isso porque a conformação física dos dispositivos móveis e a infraestrutura tecnológica pública são aspectos que extrapolam a zona de influência das instituições. Segundo Elias (2011), dada a variedade de dispositivos existentes e as limitações das estruturas de transmissão de dados, não há uma solução única para embarcar conteúdos ricos em todos os tipos de dispositivos. $\mathrm{O}$ caminho para lidar com tais obstáculos é contorná-los por meio da adaptação da mídia ao dispositivo. Esta sim é uma ação que pode ser feita pela instituição educacional.

Para contornar limitações do dispositivo e da infraestrutura, Elias (2011) propõe a implementação da mídia em formatos simples e intuitivos, e que formem pacotes de conteúdo adequados às dimensões reduzidas dos dispositivos. Isso torna a mídia mais acessível a variados modelos e pode contemplar um grupo maior de estudantes. Verifica-se, portanto, que a superação de parte das barreiras está associada ao desenho da mídia, que precisa balancear os recursos para adequar-se ao contexto tecnológico heterogêneo. $\mathrm{O}$ adequado desenho da mídia também contribui para a superação de barreiras cognitivas. Segundo Koole (2006), mídias móveis simples e intuitivas, organizadas em blocos de informações em tamanhos apropriados fazem com que os recursos cognitivos do usuário possam ser dedicados à realização da tarefa e não à superação das barreiras estabelecidas pela tecnologia. Dessa forma, pode-se também contornar, ou pelo menos diminuir, a sobrecarga cognitiva do usuário.

Algumas dificuldades contextuais, em especial aquelas relacionadas aos obstáculos de uso do AVA por meio dos dispositivos móveis e da integração do livro eletrônico aos processos de avaliação, são contornáveis na medida em que a instituição adapte seus recursos e processos às novas demandas. Adotar uma versão do AVA para dispositivo móvel é uma solução tecnicamente possível. A adaptação dos processos de EAD também é solucionável, apesar de não ser obrigatoriamente simples. Em ambos os casos, a questão depende principalmente das estratégias institucionais e de seus esforços e investimentos decorrentes.

Algumas dificuldades apontadas pelos estudantes estão relacionadas com o modo como esses percebem os dispositivos e com suas competências para fazer do dispositivo um instrumento efetivo de apoio à sua aprendizagem. Tais barreiras poderiam ser minimizadas a 
partir de ações de estímulo e orientação ao estudante, visando apoiá-lo na superação de suas resistências e na aprendizagem de novos modos de apropriar-se do conhecimento. Para Wilson e Aagard (2012), a resistência na adoção das tecnologias móveis na aprendizagem pode ser atribuída a vários fatores como a falta de conhecimento, a fobia tecnológica, a falta de tempo para explorar o potencial das tecnologias e a aversão aos riscos inerentes à implementação. Na mesma direção, Yang (2013) identificou que estudantes universitários com alto grau de habilidade para lidar com mudanças tendem a perceber mais facilmente as vantagens das iniciativas de m-learning e consequentemente têm maior propensão a aderir a elas. Estes estudantes são eficazes no enfrentamento de mudanças porque tem mais habilidades no uso das tecnologias e maior autoconfiança para superar as dificuldades enquanto ainda estão aprendendo a nova tecnologia.

Venkatesh et al. (2003), apontam que o uso educacional dos dispositivos móveis pelos estudantes é influenciado por fatores como a existência de condições ambientais facilitadoras, a influência social, as expectativas de redução de esforço e de melhoria de desempenho. Assim, para mobilizar o estudante para usar a mídia, pode-se ofertar de estímulos que enalteçam seus benefícios e apoiem a superação das barreiras percebidas. Segundo Passey (2010), uma liderança positiva perante os estudantes para demonstrar os dispositivos como recursos legítimos para a aprendizagem pode favorecer a construção de uma nova cultura e promover comportamentos de aceitação de novos modos de aprender.

Girard (2014) sugere que a superação das barreiras no uso de livros eletrônicos pode se dar por meio da negociação com o usuário quando este percebe vantagens ou oportunidades que sem o uso do dispositivo não poderia usufruir. Então, pode-se propor a troca de um recurso percebido como positivo (por exemplo, o conforto no uso do livro impresso) e negociá-lo com a oferta de recursos novos que estimulariam o interesse e uso pelo estudante (por exemplo, a disponibilidade de ferramentas de pesquisa e outros recursos interativos inexistentes no livro impresso). Motivações como a obtenção de facilidades, melhoria de desempenho, possibilidade de redução de custos, entre outras, também poderiam ser utilizadas como argumentos nesse processo de negociação e superação de barreiras.

Nos resultados encontrados evidenciou-se que os estudantes tem ainda pouco conhecimento sobre o uso de livros eletrônicos em formatos mais ricos como o EPUB. Observou-se também que há pouco conhecimento sobre recursos avançados dos programas leitores. Ao mesmo tempo, evidenciou-se que os estudantes que fizeram uso do livro eletrônico ofertado tiveram uma percepção mais positiva desta mídia do que aqueles que não fizeram uso dela. Estes indicadores apontam para uma tendência de que a familiaridade com a mídia pode fazer com que a percepção dos estudantes se transforme e se torne mais positiva. Assim, um aspecto que poderia contribuir na estratégia de disseminação da mídia móvel é o incremento das habilidades do estudante no uso avançado da tecnologia móvel. Segundo Ng (2012), a menos que haja um objetivo associado ao uso da tecnologia em sua aprendizagem é pouco provável que a maioria dos estudantes faça uso de tecnologias para fins educacionais que vão além da habitual pesquisa na internet. Estudantes costumam ter uma compreensão limitada de como a tecnologia pode apoiar sua aprendizagem. Por isso, parece oportuno também educar os estudantes para aperfeiçoar suas competências em mídias e recursos digitais, e este é um papel que a instituição educacional poderia assumir.

Uma orientação importante aos estudantes está relacionada à compreensão de sua dinâmica pessoal de estudos e do esforço cognitivo envolvido no estudo mediado pela tecnologia móvel. Terras e Ramsay (2012) destacam a dificuldade de concentração como um dos desafios da aprendizagem baseada em dispositivos móveis, pois a atenção do estudante aos estudos disputa com os variados estímulos do ambiente. Por isso, propõem que o estudante deve desenvolver habilidades para discernir o que é relevante e filtrar o que não tem valor para a aprendizagem. O estudante precisa desenvolver habilidades metacognitivas para 
resistir às interrupções do ambiente, controlar sua atenção, organizar suas tarefas e realizar seu automonitoramento. Neste sentido, verifica-se que apoiar o estudante no desenvolvimento de boas estratégias de estudo e tomada de consciência sobre seu processo de aprendizagem pode ser relevante. Este é outro papel que as instituições educacionais poderiam assumir para favorecer a adoção de mídias móveis e, em especial, do LDE.

\section{Considerações finais}

As motivações e principalmente as dificuldades relatadas pelos estudantes apontam para a importância de uma adoção cuidadosa e planejada do LDE em dispositivo móvel. De modo geral, observou-se que adoção em larga escala traz consigo a necessidade de enfrentar e gerenciar mudanças, tanto institucionais quanto individuais. Exigem também uma nova forma de pensar, tanto para construir os recursos didáticos quanto para usá-los.

Observa-se também que pode ser oportuno que o processo de mudança decorrente da inserção da mídia móvel seja gradativo, para que tanto a instituição como os estudantes possam se preparar e habituar a um novo tipo de recurso educacional. Percebe-se que para favorecer a disseminação desses recursos é oportuno o exercício de uma liderança institucional positiva em direção à mudança, que mobilize os estudantes, mas também todos os demais atores envolvidos no processo de aprendizagem.

\section{REFERÊNCIAS}

ANDERSON, T.; SHATTUCK, J. Design-based research: a decade of progress in education research? Educational Researcher, v. 41, n. 1, p. 16-25, 2012. Disponível em: http://edr.sagepub.com/content/41/1/16. Acesso em: 21 jun. 2013.

ARENAS, E.; BARR, A. The digital book in higher education: beyond the horseless carriage. In: ELETRIC DREAMS - 30th ASCILITE CONFERENCE PROCEEDINGS. Anais eletrônicos... Sydney: Ascilite, 2013. 63-67. Disponível em: http://www.ascilite.org/conferences/sydney13/program/papers/Arenas.pdf. Acesso em: 15 jul. 2015.

DESIGN-BASED RESEARCH COLLECTIVE. Design-based research: an emerging paradigm for educational inquiry. Educational Researcher, v. 32, n. 1, p. 5-8, 2003.

ELIAS, T. Universal instructional design principles for mobile learning. International

Review of Research in Open and Distance Learning, v. 12, n. 2, p. 143-156, 2011. Disponível em: http://www.irrodl.org/index.php/irrodl/article/view/965/1675. Acesso em: 23 ago. 2014.

GIRARD, A. Reader's block: a systematic review of barriers to adoption, access and use in ebook user studies. Information Research, v. 19, n. 2, 2014. Disponível em:

http://informationr.net/ir/19-2/paper624.html. Acesso em: 22 ago. 2014.

INTERNATIONAL DIGITAL PUBLISHING FORUM. EPUB 3.0 specification. Disponível em: http://idpf.org/epub/30. Acesso em: 25 abr. 2014.

KOOLE, M. L. Practical issues in mobile education. In: FOURTH IEEE INTERNATIONAL WORKSHOP ON WIRELESS, MOBILE AND UBIQUITOUS TECHNOLOGY IN EDUCATION. Anais eletrônicos... Atenas: IEEE, 2006. Disponível em: http://www.computer.org/csdl/proceedings/wmte/2006/2723/00/27230142-abs.html. Acesso em: 02 fev. 2013.

LIU, Y.; HAN, S.; LI, H. Understanding the factors driving m-learning adoption: a literature review. Campus-Wide Information Systems, v. 27, n. 4, p. 210-226, 2010. Disponível em: http://www.emeraldinsight.com/10.1108/10650741011073761. Acesso em: 15 jul. 2012. 
MATTA, A.; SILVA, F. P. S.; BOAVENTURA, E. M. Design-based research ou pesquisa de desenvolvimento: metodologia para pesquisa aplicada de inovação em educação do século XXI. Revista da FAEEBA: Educação e Contemporaneidade v. 23, n. 42, p. 23-36, 2014. Disponível em: http://www.revistas.uneb.br/index.php/faeeba/article/view/1025. Acesso em: 14 jul. 2014.

NG, W. Can we teach digital natives digital literacy? Computers \& Education, v. 59, n. 3, p. 1065-1078, 2012. Disponível em: http://www.sciencedirect.com/science/article/pii/S0360131512001005. Acesso em: 14 jul. 2014.

PASSEY, D. Mobile learning in school contexts: can teachers alone make it happen? IEEE Transactions on Learning Technologies, v. 3, n. 1, p. 68-81, 2010. Disponível em: http://ieeexplore.ieee.org/lpdocs/epic03/wrapper.htm?arnumber=5306066. Acesso em: 30 mai. 2013.

SANTAELLA, L. A ecologia pluralista da comunicação: conectividade, mobilidade e ubiquidade. São Paulo: Paulus, 2010.

SHARPLES, M.; TAYLOR, J.; VAVOULA, G. A theory of learning for the mobile age. In: ANDREWS, R. ; HAYTHORNTHWAITE, C. The sage handbook of e-learning research. Londres: Sage, 2007. Disponível em: http://www.uk.sagepub.com/refbooks/Book228475. Acesso em 03 fev. 2013.

TERRAS, M. M.; RAMSAY, J. The five central psychological challenges facing effective mobile learning. British Journal of Educational Technology, v. 43, n. 5, p. 820-832, 2012. Disponível em: http://onlinelibrary.wiley.com/doi/10.1111/j.1467-8535.2012.01362.x/pdf. Acesso em: 17 mai. 2013.

TRAXLER, John. Current state of mobile learning. In: ALLY, M (Org.). Mobile learning: transforming the delivery of education and training. Edmonton: AU Press, Athabasca University, 2009. Disponível em: www.aupress.ca/index.php/books/120155. Acesso em: 12 fev. 2013.

VENKATESH, V.; MORRIS, M. G.; DAVIS, G. B.; DAVIS, F. D. User acceptance of information technology: toward a unified view. MIS Quarterly, v. 27, n. 3, p. 425-478, 2003. Disponível em: http://www.jstor.org/stable/30036540. Acesso em: 14 mar. 2013.

WILSON, M.; AAGARD, S. D. Exposing the gap between what is possible and what is acceptable: how m-learning can make a contribution to sonography education. Journal of Diagnostic Medical Sonography, v. 28, n. 4, p. 202-206, 2012. Disponível em: http://jdm.sagepub.com/cgi/doi/10.1177/8756479312444349. Acesso em 19 ago. 2012.

YANG, S. An emerging learning mode: tan emerging learning mode: the effects of instrumental beliefs, extrinsic influences and personal traits on undergraduate students' adoption of mobile learning. International Journal of Digital Content Technology and its Applications, v. 7, n. 5, p. 697-706, 2013. Disponível em: http://www.aicit.org/jdcta/global/paper_detail.html?jname=JDCTA\&q=2776. Acesso em: 18 abr. 2013. 